\title{
Zustandsbasierte Optimierung der Kalibrierzyklen von Prozesssensoren mittels adaptiver Monte Carlo-Simulation
}

\author{
Dipl.-Wirtschaftsing. Hans Joachim Fröhlich ${ }^{1}$, Dr. Dimitri Vaissière ${ }^{2}$ \\ Endress+Hauser Flowtec AG, Kägenstr. 7, 4153 Reinach, Schweiz \\ ${ }^{2}$ Endress+Hauser Consult AG, Colmarer Str. 6, 79576 Weil am Rhein, Deutschland \\ hans-joachim.froehlich@flowtec.endress.com
}

\begin{abstract}
Zusammenfassung
Das Konzept der vorbeugenden Wartung hat insbesondere in regulierten Prozessindustrien den Ansatz fester Kalibrierzyklen für Prozesssensoren zementiert. Diese werden gegenwärtig in Abhängigkeit ihrer Kritikalität und einer statisch bewerteten Fehlerwahrscheinlichkeit pro Messstelle festgelegt. Insbesondere Verlängerungen sind empirisch schwer zu begründen und regulatorisch schwer umzusetzen. Dem Betreiber entgeht dabei die Möglichkeit zur Optimierung des Kalibriermanagements aufgrund realer Drift-Risiken. Der hier vorgestellte Ansatz zeigt die Möglichkeiten auf, mit Hilfe von Monte Carlo-Simulation zu einer Risikobewertung zu gelangen, welche die reale, individuelle Kalibrierhistorie jeder Messstelle mit einbezieht. Daraufhin können Empfehlungen zur Anpassung von Kalibrierzyklen mit Rücksicht auf Betriebskosten, Anlagenverfügbarkeit und Risikominimierung gegeben werden. Werden zusätzlich Condition Monitoring-Daten hinzugezogen, kann die Methode weiter verfeinert werden. Der mithin adaptive Simulationsansatz führt dazu, dass der Schwerpunkt des Prüfaufwands sich dorthin verlagert, wo es sinnvoll ist, nämlich an die Messstellen mit hohem Drift-Risiko und hoher Kritikalität. Der Ansatz ist, was seinen Ressourcenbedarf betrifft, auch im Hinblick auf das Informationsmanagement konsistent, d.h. der Aufwand für die Datenerfassung, Übertragung und Verarbeitung wird dort konzentriert, wo er die betriebliche Optimierung am effektivsten unterstützt.
\end{abstract}

Keywords: Sensorwartung,Monte Carlo-Simulation,Risikobewertung,Optimierung von Kalibrierzyklen,Prozesssensoren 4.0.

\section{Zielkonflikt der Sensorwartung in regulierten Produktionsprozessen}

Um die Sicherheit, die Verfügbarkeit und die Wirtschaftlichkeit von prozesstechnischen Anlagen zu gewährleisten, hat sich in den vergangenen Jahrzehnten die vorbeugende gegenüber der reaktiven Wartung als normativer Ansatz etabliert.

Bereits ein Qualitätsmanagement nach ISO 9001 verlangt vom zertifizierten Betreiber die regelmäßige Prüfung der Funktionsfähigkeit seiner Systeme und Anlagen sowie deren Komponenten. Hierzu zählen auch die in den Produktionsprozessen genutzten Messgeräte, welche periodisch einer den internationalen Regeln der Metrologie entsprechenden Prüfung mittels Verifikation oder Kalibrierung zu unterziehen sind [1]. Lässt diese Norm dem Betreiber noch manchen Spielraum in der Umsetzung, geben speziellere Vorschriften nicht selten exakte, arbiträre Prüfintervalle vor.
Das Zielsystem für das Kalibriermanagement in verfahrenstechnischen Prozessen umfasst, insbesondere in regulierten Umgebungen, neben wirtschaftlich relevanten Größen wie Anlagenverfügbarkeit und Wartungsaufwand somit vor allem die Vermeidung von Risiken im Hinblick auf die Produktqualität.

Auch die Qualitätssicherung unter GMP sieht eine periodische Validierung aller Messstellen im Produktionsprozess vor [2].

Der Wartungsansatz ist ebenfalls vorbeugend, d.h. es werden, abhängig von der Kritikalität einer Messstelle für die Produktqualität, feste Intervalle vorgegeben, nach denen die Kalibrierung des Sensors vorzunehmen ist. Dieser Ansatz wirkt nicht nur für sich bereits kostentreibend. Er bringt es darüber hinaus mit sich, dass die überwiegende Anzahl der Sensoren bei der Kalibrierung als unverändert, manche jedoch schon als fehlerhaft befunden werden. Für erstere Geräte stellt sich die kostspielige Kalibrierung somit als überflüssig, für die zweite als unwirksam heraus, was 
sodann aufwändige Maßnahmen zur weiteren Risikoabwehr nach sich zieht. Im Extremfall werden teure Rückrufaktionen erforderlich. Kann eine Nachlieferung einwandfreier Ware nicht zeitnah erfolgen, entgehen dem Hersteller Marktanteile und Deckungsbeiträge, Produkt- und Unternehmensmarke können in ihrem Wert nachhaltig beeinträchtigt werden.

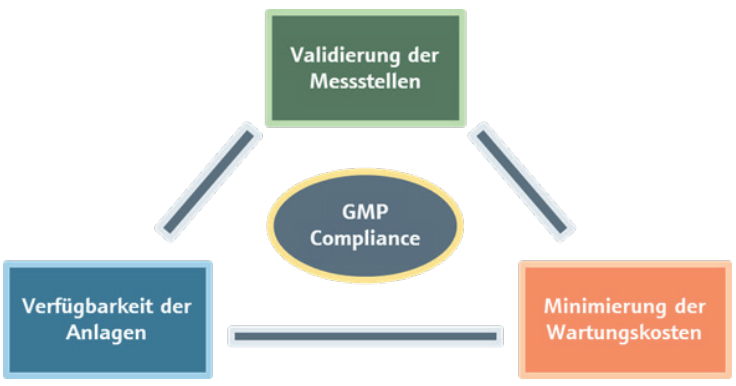

Abb. 1: Zielsystem für das Kalibriermanagement unter GMP, Endress+Hauser 2017

Werden daraufhin Kalibrierzyklen verkürzt, verbessert sich zwar die Risikosituation, es verschlechtert sich jedoch die Zielerreichung im Hinblick auf Anlagenverfügbarkeit und Wartungsaufwand. Werden die Kalibrierzyklen dagegen verlängert, erhöht sich (zumindest im Planansatz) die Wirtschaftlichkeit, es wächst jedoch das Risiko und mithin der zu erwartende Aufwand für Nachbesserungen.

Somit kann festgehalten werden, dass die vorbeugende Wartung keine befriedigende Optimierung im Hinblick auf die grundsätzlich in Konflikt stehenden Zielgrößen liefert.

\section{Zweidimensionaler Ansatz für die zielgerechte Wartung von Sensorsystemen}

Neben der bereits angesprochenen Kritikalität von Messstellen im Hinblick auf die Produktoder Prozessqualität ist, als zweite Dimension, das Risiko des Einflusses von Prozess- oder Umgebungsbedingungen auf die Funktionalität bzw. die Messleistung von Sensorsystemen mit einzubeziehen. Diese beiden voneinander prinzipiell unabhängigen Input-Dimensionen bestimmen, für jede Messstelle individuell, die gebotene Intensität der Sensorwartung. Über die gesamte installierte Basis an einem Produktionsstandort verlagert sich der Schwerpunkt der Wartung auf Messstellen mit hoher Kritikalität und hohem Risiko für SensorDrift. Wird die Sensorwartung entsprechend betrieblicher oder regulatorischer Vorgaben mittels Kalibrierung durchgeführt, erweist es sich gewöhnlich als notwendig, an solchen Messstellen hierfür die Frequenz zu erhöhen, während Sensoren an Messstellen mit geringerer Kritikalität oder geringerem Drift-
Risiko entsprechend weniger häufig zu kalibrieren sind. Während die Kritikalitätsbewertung für die Betreiber von Anlagen unter GMP bereits Routine ist, erweist sich die Bewertung des Risikos von Sensordrift als neu und schwierig.

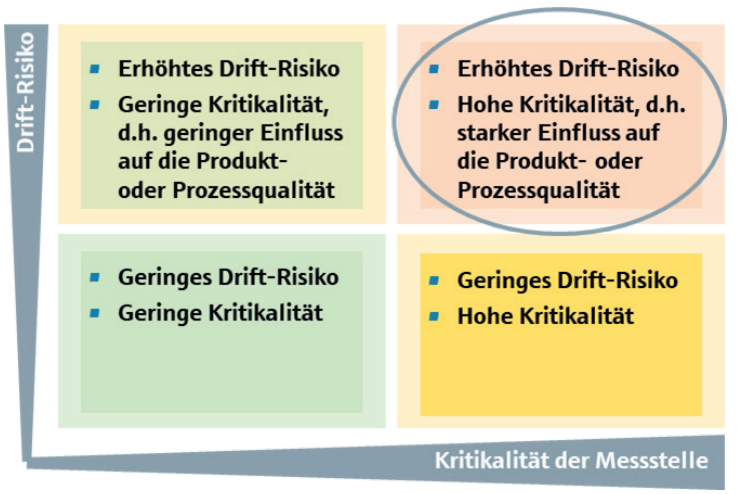

Abb. 2: Ausrichtung der Sensorwartung auf Messstellen mit hohem Risiko und hoher Kritikalität, Endress+Hauser 2017

Gewöhnlich werden wiederum statische Methoden zur Bewertung des Risikos von Sensorversagen herangezogen [2].

$\mathrm{Zu}$ unterscheiden sind hierbei spontan auftretende transiente Störfaktoren, wie eine erhöhte Luft- oder Gasbeladung von flüssigen Messstoffen, etwa durch defekte Dichtungen oder Ventile, von längerfristig wirkenden Faktoren, wie etwa Alterung von Elektronikbauteilen, sowie Ablagerungen oder Korrosion in sensiblen Bereichen der Sensorik. Während die erste Kategorie faktisch keine Verschlechterung des Sensorzustandes nach sich zieht und durch gezieltes Condition Monitoring ggf. als Störung des Prozesses zu detektieren ist, muss die zweite Art des Prozesseinflusses hinsichtlich ihres allmählichen Einflusses auf die Messleistung interpretiert werden.

Wesentliches Kriterium zur Interpretation ist hierbei das Risiko, dass der für die Messstelle spezifizierte maximal zulässige Fehler MPE (maximum permissible error) überschritten wird. Um eine sinnvolle Einschätzung dieses Risikos für eine konkrete Messstelle vornehmen zu können, wäre es naheliegend, auf eine Historie an vergleichbaren Messstellen zurückgreifen zu können. In der Praxis wird dies jedoch ausnahmsweise nur dann möglich sein, wenn eine signifikante Anzahl an Messstellen über einen hinreichend langen Zeitraum betrachtet wird.

Hierzu wären aktuelle Kalibrierdaten einer hinreichend großen Stichprobe an Messstellen auszuwerten, welche über ihre Einsatzdauer hinweg mit Sicherheit vergleichbaren 
Umgebungs- und Prozessbedingungen ausgesetzt sind - alle Durchflussmessgeräte etwa, die zur Überwachung von Pumpen des gleichen Typs mit vergleichbarer Laufleistung eingesetzt werden.

Es liegt auf der Hand, dass in der Definition und Abgrenzung solcher Stichproben erhebliche Unsicherheiten liegen, weshalb sich für die Praxis nur in wenigen Ausnahmen sinnvolle Handlungsanweisungen hieraus ergeben. Bevorzugt wird stattdessen eine Einteilung in Risikoklassen, die im Laufe der Nutzung, so lange keine wesentlichen Störfälle verzeichnet werden, nicht wieder infrage gestellt wird.

\section{Simulation als Ausweg aus dem Mangel an signifikanten Historien zur Sensorwartung}

Grundsätzlich lassen sich jedoch die typischen Belastungsfälle von Messgeräten im Prozess gut in stochastischen Modellen abbilden, um ihren allmählichen Einfluss auf die Genauigkeit des Sensorsystems zu simulieren.

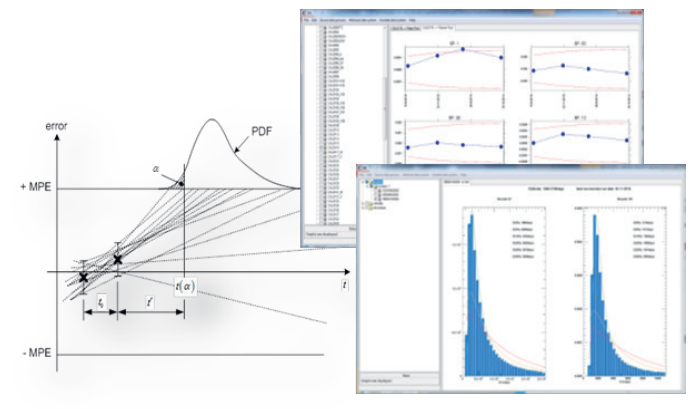

Abb. 3: Simulation von Prozesseinflüssen auf die Messleistung von Sensoren, Endress+Hauser 2017

Aus einer endlichen Auswahl an möglichen Belastungsszenarien werden die für eine bestimmte Prozessmessstelle relevanten Möglichkeiten ausgewählt und in ihrer Wirkung über die Zeit in einer stochastischen Verteilung abgebildet. Es folgt eine große Anzahl an Simulationen im Hinblick auf die Geschwindigkeit des Fortschreitens von Sensordrift, welche die Auswirkungen der Belastungsszenarien auf die Messleistung des Sensorsystems überführt.

Hiermit werden empirische Dichtefunktionen für die Wahrscheinlichkeit ermittelt, mit welcher sich die Möglichkeit eines Überschreitens von zulässigen Messfehlern vorausschauend bewerten lassen. Hieraus lassen sich somit risikobasierte Empfehlungen für die Häufigkeit von Rekalibrierungen herleiten, um die
Wahrscheinlichkeit einer unzulässig hohen Sensordrift unterhalb eines tolerierten Niveaus zu halten.

Dies allein wäre schon hinreichend, um eine wie oben beschriebene, unter Risikoaspekten sinnvolle Differenzierung der Messstellen zu Zwecken des Kalibriermanagements zu unterstützen. Ihr volles Potenzial entfaltet die Methode jedoch erst, wenn die reale Drift an jeder Messstelle mit einbezogen werden kann. Liegen nun also nach der üblichen Kalibrierung vor Inbetriebnahme sowie einer ersten Rekalibrierung im Betrieb zwei Datenpunkte für die Messleistung des betreffenden Sensors vor, kann daraufhin eine erste Anpassung der simulierten Sensordrift erfolgen. Diese liefert mit einem definierten Konfidenzniveau eine Aussage zum empfohlenen nächsten Kalibrierintervall. Da sich diese Empfehlung im Laufe der Zeit immer wieder den Ergebnissen erneuter Rekalibriervorgänge anpasst, kann von einer adaptiven Simulation gesprochen werden. Die Möglichkeiten dieses Ansatzes können nun maximiert werden, wenn nicht nur die aktuellsten Datenpunkte der Kalibrierung in Betracht gezogen werden, sondern weitere aussagekräftige Parameter aus dem Condition Monitoring der Sensorsysteme hinzugezogen werden.

Moderne Messsysteme bieten mittels ihrer Selbstüberwachungsfunktion [3] kontinuierlich Zustandsparameter an, die Aufschluss über die Auswirkung von Prozessbedingungen auf die Messleistung geben. Dies hat zweierlei Vorteile: zum einen können die Informationen in sinnvollen Zeitabständen in die Bewertung des Drift-Risikos mit einfließen. Monte CarloSimulationen werden entsprechend häufiger durchgeführt, ihre Aussagekraft beträchtlich erhöht. Zum anderen kann das Condition Monitoring bereits für sich - d.h. zwischen den erneuten Simulation - wichtige Aussagen über den Sensorzustand liefern und mithin etwa im Falle einer unvorhergesehen auftretenden Drift vorausschauend Hinweise auf eine notwendige vorgezogene Rekalibrierung geben.

Mit diesem vom aktuellen Zustand der Messsysteme abhängigen Ansatz kann die Optimierung der Wartungszyklen nicht nur alle oben genannten Zielgrößen simultan berücksichtigen, sondern im Lebenszyklus der Anlage ebenfalls adaptiv erfolgen. $\mathrm{Da}$ die Informationen über den Prozess und seine Messsysteme im Laufe der Zeit immer weiter vervollständigt werden können, ist der Betrieb in der Lage, seine Zielgrößen Schritt für Schritt zu verbessern. Für Messstellen mit hohem Wartungsbedarf werden das kostengünstige Condition Monitoring und die aufwändigere Rekalibrierung aufeinander abgestimmt. Für 
Messstellen mit geringer Kritikalität und geringem Drift-Risiko kann die Rekalibrierung evtl. ganz entfallen.

Der Ressourceneinsatz zur Validierung der Prozessmessgeräte kann somit immer weiter auf die Punkte konzentriert werden, an denen er tatsächlich am meisten Nutzen stiftet, nämlich an Messstellen, die sowohl eine hohe Bedeutung für die Produktqualität aufweisen, als auch tatsächlich einem erhöhten akuten Risiko von Abweichungen in der Messleistung ausgesetzt sind.

\section{Big Data oder Small Data? - Betrachtungen zum Informationsmanagement}

Die Zyklen zur Erfassung geeigneter „LiveDaten" aus dem Prozess zwecks Optimierung der Sensorwartung können nun den gleichen Richtlinien folgen, wie die Kalibrierintervalle selbst: je geringer Drift-Risiko und Kritikaliltät eingestuft werden, umso seltener werden aktuelle Condition Monitoring-Daten benötigt und umso seltener ist eine erneute Simulation für die betreffende Messstelle erforderlich. Was den Aufwand zur Risikobewertung betrifft, folgt der Ansatz somit konsistent seiner Logik einer bedarfsgerechten Ressourcenallokation. Hieraus ergibt sich ein bemerkenswerter Bruch mit dem vorherrschenden Paradigma, welches sich für Prozessüberwachung und Optimierung im Kontext von Industrie 4.0 festgesetzt hat, nämlich mit dem „Big Data“-Begriff.

Unter „Big Data“" wird hierbei die Verarbeitung großer Datenmengen verstanden, die aus dem Feld gewonnen werden, um unter Anwendung statistischer Entscheidungsmodelle konkrete Handlungsempfehlungen für differenzierte Anwendungsfälle zu gewinnen. Bedeutung hat dieser Ansatz durch die immense Ausweitung der Nutzung des Internets für Transaktionen zwischen Anbietern bestimmter Leistungen und Verbrauchern gewonnen (B2C). Neben der Marktsegmentierung zwecks differenzierter Kommunikationsmaßnahmen ist aktuell die personalisierte Angebotsformulierung in das Zentrum des Interesses gerückt [4]. Aufgrund von Konsumprofilen sollen möglichst jedem Kunden bedarfsgerechte Angebote unterbreitet werden - jüngst auch unter Berücksichtigung der kalkulierten individuellen Zahlungsbereitschaft (personalized pricing).

Derartige Vorstellungen sind für das Thema Industrie 4.0 (B2B) von vielen Beitragenden ohne ersichtliche Reflektion übernommen und - im Hinblick auf ihre Zweckmäßigkeit unzureichend kommentiert - mit Konzepten wie etwa der "Schwarmintelligenz" von Sensoren kombiniert worden [5].

Kritisch zu sehen ist hierbei zunächst die Übertragungskapazität der Feldnetzwerke, die primär für Zwecke der Prozessautomatisierung sowie der laufenden Qualitätssicherung ausgelegt sind - und nicht zur Akquisition großer Mengen an Zustandsdaten rund um die Assets selbst.

Auch wenn durch leistungsfähige Ethnetbasierte Protokolle, wie etwa PROFINET oder EtherNet/IP, eine beträchtliche Bandbreite mit sinnvollen Reserven installiert ist, kann nicht davon ausgegangen werden, dass für die Zwecke des Condition Monitoring von Anlagen und deren Komponenten kurzfristig genügend Bandbreite vorhanden ist. Bis die installierte Basis zu neuen Standards hin migriert, wie etwa den Namur Open Architecture-Ansatz für Prozesssensoren 4.0 [5], bleibt der Zugang über das Feldnetzwerk ein limitierender Faktor. Dies kann u.a. einen erheblichen Versatz bei der Datenübermittlung zur Konsequenz haben, so dass zeitlich an sich zusammengehörige Datensätze auseindander gerissen werden und dissynchronisiert in den empfangenden Datenbanken eintreffen.

Sich daraus ergebende Betrachtungsfehler lassen sich statistisch mit nur mit Hilfe noch grösserer Datenmengen wieder korrigieren.

Umfängliche Konzepte mit modernen EdgeGateways an zahlreichen dezentralen Zugangsstellen halten dem prioritären Automatisierungssystem zwar den Rücken frei, müssen sich jedoch stets noch der begrenzten Kapazität auf den letzten Metern zu den Messstellen fügen. Für die Praxis durchaus verfügbare Messstellenadapter führen zum Aufbau von Funknetzwerken, wie etwa auf WirelessHART-Basis, die ebenfalls in ihrer Bandbreite begrenzt sind. Ihr hoher Investitionsaufwand hält viele Betreiber davon $\mathrm{ab}$, sich mit dieser Materie zu befassen.

Zum anderen ist unter diesen Gesichtspunkten auch der Speicher- und Rechenaufwand kritisch zu sehen, den Big Data-Ansätze zur Wartungs- und Prozessoptimierung mit sich bringen.

Die hier vorgestellte Methode belegt nun vor allem in der Umsetzung in Pilotanwendungen ihren Pragmatismus im Umgang mit den gegenwärtig verfügbaren Ressourcen. Was den Kapazitätsbedarf zur Datenübertragung betrifft, kann hier für die relevanten Anwendungsfälle durchaus von einem „Small Data"-Ansatz die Rede sein. Da sich Ablagerungs- oder Korrosionsvorgänge gewöhnlich über Wochen, Monate oder Jahre hinziehen, ist eine Zustandsüberwachung im Bereich von Sekunden oder Minuten kaum sinnvoll. Frequenzen zur Datenerfassung und Übertragung von einigen Stunden für jede Messstelle können für die meisten Zwecke als vollkommen ausreichend betrachtet werden. 
Wie oben beschrieben, kann die Anforderung solcher Daten in Abhängigkeit der Risikobewertung optimiert werden

Die Monte Carlo-Simulation gehört zwar zu den aufwändigeren Simulationsmethoden, insgesamt ergibt sich jedoch auch für eine installierte Basis von einigen hundert bis einigen tausend Geräten kein Aufwand, der nicht von einigen wenigen Rechnerstationen bewältigt werden kann.

\section{Praktische Umsetzung}

Zum Proof-of-Concept wurde dieses Konzept im Pilotmaßstab in Biotechnologieanlagen sowohl eines großen europäischen Herstellers von Arzneimitteln, wie auch im Technikum des irischen NIBRT (National Institute for Bioprocessing, Research and Training) anhand verschiedener Messgeräte mit umfassender Fähigkeit zur Selbstüberwachung konkretisiert. Die Monte Carlo-Simulation zur Ermittlung des aktuellen Drift-Risikos erfolgt mit Hilfe eines proprietären Modells von Endress+Hauser.

Àhnliche „Leuchtturmprojekte" sind mit grossen Herstellern chemischer Produkte im Gang.

Auch wenn die in den Pilotanlagen eingestellten Szenarien einen Prozess mit hohem Anspruch an die Stabilität der Messstellen verkörpern, wird es einige Zeit in Anspruch nehmen, bis der Praxisnachweis für die Vorteilhaftigkeit der neuen Methode mit Blick sowohl auf die Risikobewertung, wie auch in wirtschaftlicher Hinsicht erbracht ist.

Eine für den Betreiber außerordentlich hilfreiche Erkenntnis konnte indessen bereits zusätzlich gewonnen werden: Ist der Einfluss des Prozesses auf die Messunsicherheit des Sensorsystems wenigstens seiner Natur nach bekannt, was für die meisten Messstellen ohne weiteres vorausgesetzt werden kann, lassen sich die Aussagen des Condition Monitoring aufgrund der unterschiedlichen Ausrichtung der zugrundeliegenden Parameter zum einen in Sensor-Drift, zum anderen in Prozess-Drift einteilen. Sind zudem Condition MonitoringDaten von aktiven Anlagenkomponenten wie Pumpen oder Ventilen verfügbar, lässt sich die Aussagefähigkeit weiter erhöhen. Gerade in der Inbetriebnahme-Phase neuer Anlagen kann diese Unterscheidung dem Betreiber wichtige Erkenntnisse über die Ursachen von Inkonsistenzen im Prozess liefern und beträchtliche Zeit bei der Fehlersuche und Behebung sparen. Das gleiche gilt über die gesamte Nutzungsdauer für die Identifikation und Behebung von Störszenarien.

\section{Literaturnachweis}

[1] ISO/EN/DIN 9001:2008 Kapitel 7.6 a) Lenkung von Überwachungs- und Messmitteln; bzw. ISO/EN/DIN 9001:2015, Kapitel 7.1.5.2 a) Rückführbarkeit von Messwerten

[2] Current Good Manufacturing Practices, 2011, US Department of Health and Human Services, Food and Drug Administration

[3] Namur-Empfehlung 107 (NE 107), Selbstüberwachung und Diagnose von Feldgeräten

[4] Gabler Wirtschaftslexikon, Online-Version, 2017

[5] Technologie-Roadmap „Prozesssensoren 4.0“, 2015, VDI/VDE-Gesellschaft Mess- und Automatisierungstechnik 